\title{
Venomics
}

\section{Tiergifte als Quelle neuartiger Bioressourcen}

TIM LÜDDECKE, BJÖRN M. VON REUMONT

LOEWE ZENTRUM FÜR TRANSLATIONALE BIODIVERSITÄTSGENOMIK, FRAUNHOFER

INSTITUT FÜR MOLEKULARBIOLOGIE UND ANGEWANDTE ÖKOLOGIE UND INSTITUT

FÜR INSEKTENBIOTECHNOLOGIE, UNIVERSITÄT GIESSEN

\section{Toxins evolved convergently in all major animal groups for predation, defense or competition. They are either actively employed with a de- livery apparatus as venoms, or passively secreted as poisons. The evo- lutionary arms race between toxicity and resistance in predators and their prey optimised effective toxin cocktails, which thus represent powerful candidates for translational research.}

DOI: $10.1007 / \mathrm{s} 12268-020-1482-3$

(C) Die Autoren 2020

Die Verwendung von Giften zum Beutefang, der Verteidigung oder der Konkurrenzvermeidung ist im Tierreich weit verbreitet. Durch konvergente Evolution haben alle bekannten Phyla giftige Vertreter hervorgebracht [1]. Diese haben zwei verschiedene Formen der Giftigkeit entwickelt [2]: Aktiv giftige Tiere, wie Spinnen oder Schlangen, injizieren ihr aus Polypeptiden zusammengesetztes Gift über einen Stich oder Biss in ihr Gegenüber (Abb.1). Im Gegensatz dazu gelangen die überwiegend aus niedermolekularen Stoffen, z. B. Alkaloiden, bestehenden Gifte von passiv giftigen Tieren wie Amphibien oder Schmetterlingen durch Resorption an ihren Wirkungsort (Abb. 1).

Tiergifte beinhalten viele unterschiedliche Biomolekülklassen und gehören zu den komplexesten biochemischen Systemen des Tierreichs. Im Laufe der Evolution unterliegen sie einem evolutionären Wettrüsten und werden somit stetig hinsichtlich ihrer Funktion optimiert. Als Resultat beinhalten sie hochpotente Toxine mit erstaunlicher Selektivität und großer Wirksamkeit, was sie zu exzellenten Ausgangsstoffen für die Entwicklung neuartiger Therapeutika, industriell nutzbarer Enzyme oder Bioinsektizide macht. Diese biochemische „Schatztruhe“ ist daher zunehmend im Fokus der Forschergemeinschaft und einige Wirkstoffe wurden ausgehend von Tiergiften bereits entwickelt [3]. Überdies sind Gifte und ihre Bestandteile exzel- lente Modellsysteme, um die Evolution von Genen und Proteinfunktionen im Tierreich besser zu verstehen.

\section{Das junge Forschungsfeld der Venomics}

Traditionellerweise erfolgte die Studie von Tiergiften mittels dekomplexierender Technologien, wie Flüssigchromatographie. Die Rohgifte der zu untersuchenden Arten wurden über verschiedenste Chromatographiesysteme in ihre Komponenten fraktioniert. Die Struktur der gewonnenen Komponenten wurde dann mittels Edman-Abbau, NMRoder Massenspektrometrie untersucht und ihre Biochemie in verschiedenen Assays aufgeklärt [4]. Diese Strategien halfen, die Gifte zu entschlüsseln - insbesondere von Schlangen, großen Spinnen und Kegelschnecken.

Leider sind solche Ansätze ausgesprochen probenintensiv, weshalb bisher große Giftmengen als Ausgangsmaterial notwendig waren. Als Konsequenz war es durch stark limitierte Giftmengen bislang nahezu unmöglich, die Gifte der abertausenden, sehr kleinen wirbellosen Tierarten, wie z. B. einheimischen Spinnen oder Insekten, detailliert zu untersuchen. Seit kurzem erlauben die rasant voranschreitenden Entwicklungen im Bereich der Sequenziertechnologien, Biotechnologie und vor allem der Massenspektrometrie durch ihre gesteigerte Sensitivität und Präzision auch die Analyse von Giften dieser kleineren Organismen. Die Grundlage solcher Studien bildet das Feld der Proteomics, ergänzt durch Transkriptom- und Genomanalysen. Die integrative Anwendung solcher Omics-Technologien zur Untersuchung von Giftsystemen wird - in Anlehnung an das englische Wort für Tiergift (venom) - als Venomics bezeichnet. Sie stellt in dieser synthetischen, in Abbildung 2 zusammengefassten, Form ein sehr junges Forschungsfeld dar, auf dem unsere Gruppe aktiv ist $[5,6]$.

\section{Den Giften vernachlässigter Arten auf der Spur}

Mittels moderner Venomics entschlüsselten wir jüngst erfolgreich die Giftkomposition einiger kleinen Arten erfolgreich und erlangten verblüffende Einsichten bezüglich ihrer Zusammensetzung und Bioaktivität.

Die Giftkomponenten der parasitoiden Wespe Pimpla turionellae beispielsweise waren bislang weitgehend unbekannt [7]. Wir konnten zeigen, dass das Gift von $P$. turionellae eine Reihe von vermutlich neurotoxischen Peptiden mit Disulfidbrücken in Knotenstruktur beinhaltet. Analoga dieser Klasse wurden bis jetzt hauptsächlich in Vogelspinnen und Kegelschnecken identifiziert und sind interessante Wirkstoffkandidaten [3]. P. turionellae nutzt Insekten als Wirte und als Nahrung für ihre Jungtiere. Im Wachsmotten-(Galleria mellonella)-Modell konnte nachgewiesen werden, dass das Gift von P. turionellae in der Lage ist, mittels epigenetischer Reprogrammierung die Immunantwort ihres Wirts zu unterdrücken und somit die Überlebenschancen ihrer abgelegten Larven zu erhöhen [8]. Die Toxine in dieser Gruppe sind daher einerseits extrem interessant für pharmakologische oder landwirtschaftliche Anwendungen, andererseits erlaubt eine detailliertere Kenntnis dieser Pharmakodynamik im Insekt ein besseres Verständnis der Evolution von Parasit-WirtBeziehungen.

Eine rezente Analyse des Gifts der Wespenspinne Argiope bruennichi zeigt, dass auch in Spinnengiften viel Neues zu entdecken ist [9]. 
AKTIV GIFTIGE ARTEN

(mit Beispielen für Giftapparate)

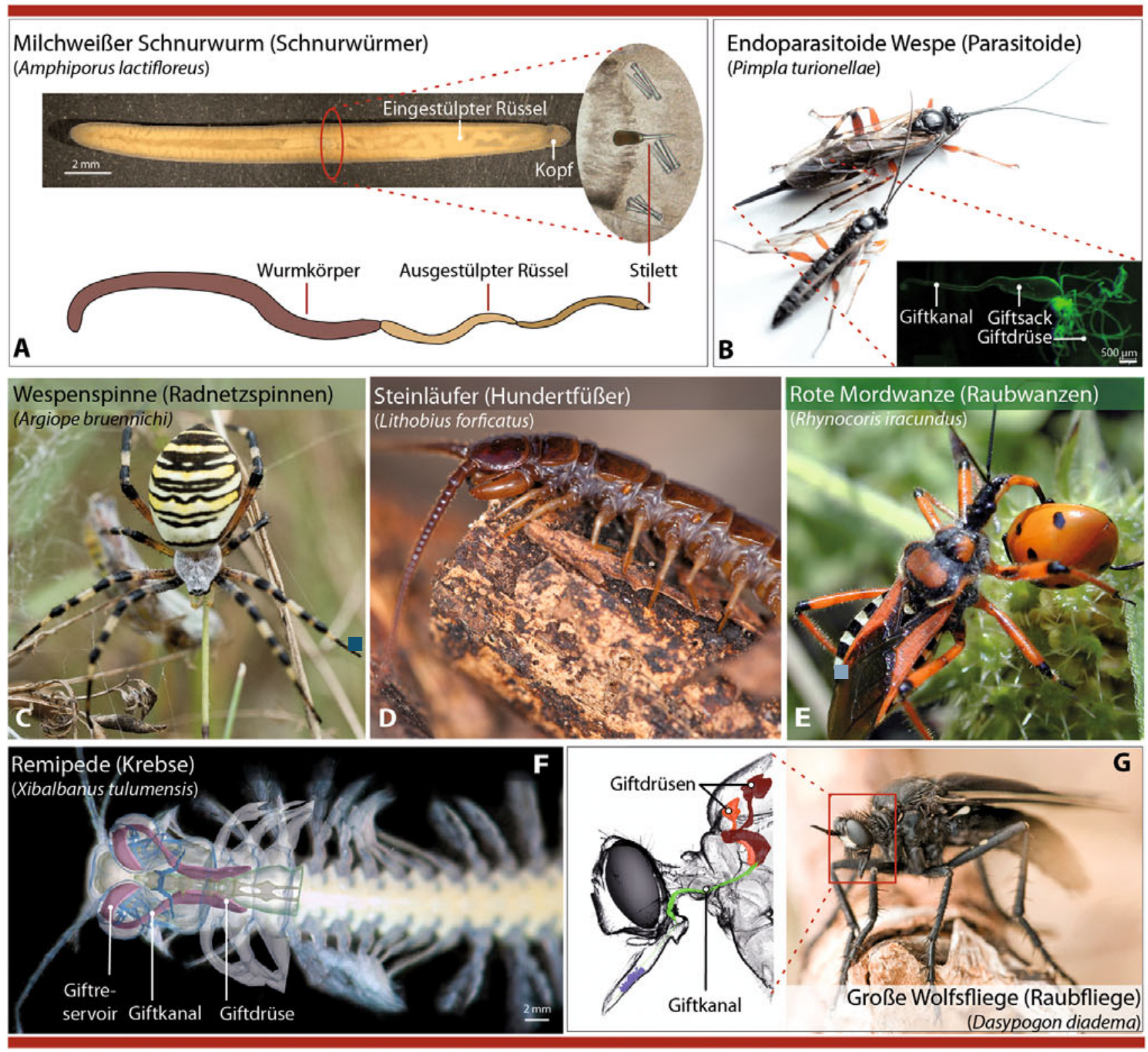

PASSIV GIFTIGE ARTEN

(Toxine unspezifisch über Hautdrüsen abgesondert)

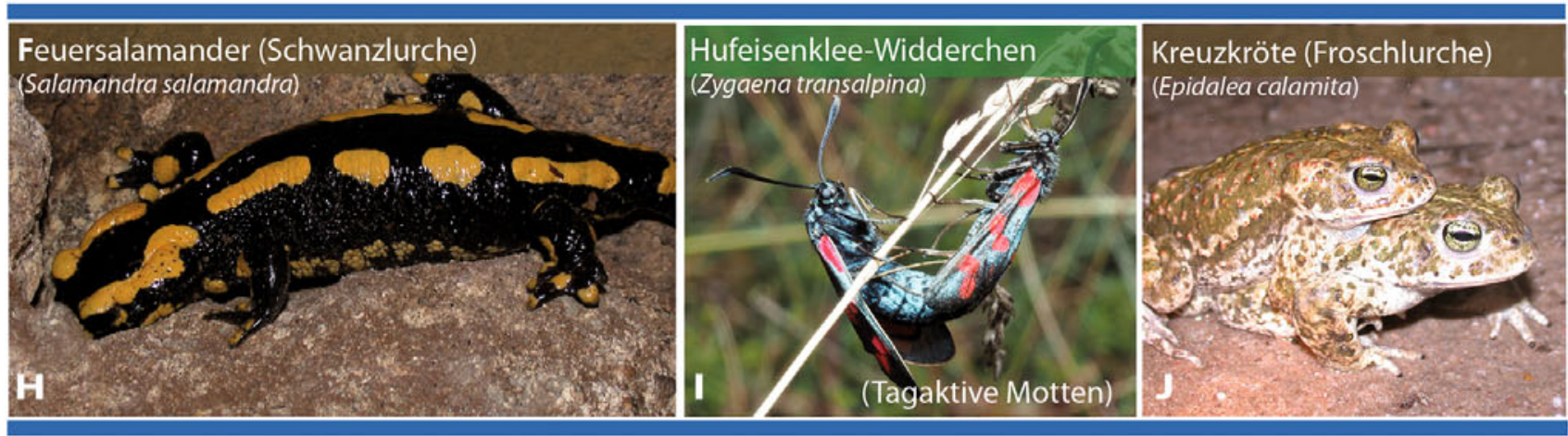

$\Delta$ Abb. 1: Auswahl aktiv giftiger Tierarten und einige ihrer komplexen Giftapparate im Vergleich zu passiv giftigen Arten. 


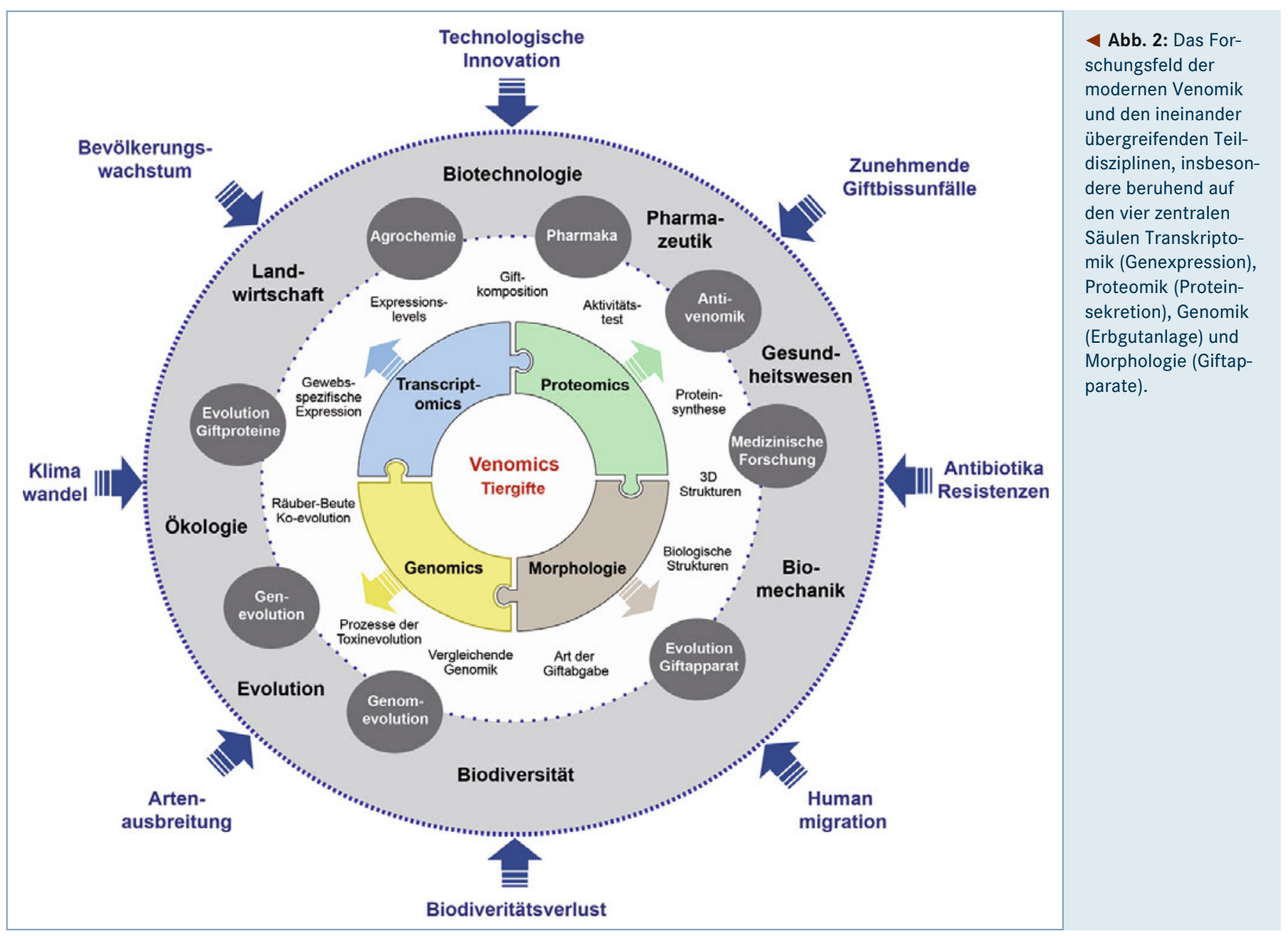

Im Gegensatz zu den chemisch hochkomplexen Giften der bisher untersuchten Arten ist das Gift der Wespenspinne recht einfach aufgebaut. Während Spinnengifte oft bis zu 1.000 Komponenten besitzen, zeigt das der Wespenspinne nur etwa 50 Bestandteile. Klassischerweise wird davon ausgegangen, dass in Spinnengiften kleine neurotoxische Peptide die Hauptkomponenten sind. Im Gift der Wespenspinne spielen diese jedoch eine eher untergeordnete Rolle, während hochmolekulare Proteinbestandteile dominieren. Ein Grund könnte sein, dass Wespenspinnen sich an eine netzbasierte Jagdstrategie anpassten und dem Giftapparat nur eine Nebenfunktion zukommt. Da sowohl Spinnseide als auch Gifte energetisch kostspielig sind, fiel eine Vielzahl der nun überflüssigen Giftkomponenten vermutlich negativer Selektion zum Opfer und ging verloren. Abschließend deckten wir auch auf, dass Wespenspinnengift dennoch einige neuartige Toxine enthält, die strukturelle Ähnlichkeit mit Neuropeptiden aus Insekten aufweisen.
Jüngst untersuchten wir in Kooperation mit dem Senckenberg Museum in Frankfurt a. M. und der Universität Bonn im Rahmen des hessischen LOEWE Forschungprojekts Translationale Biodiversitätsgeomik (LOEWE TBG) die Gifte von marinen Schnurwürmern oder Nemertea [10]. Die Art Amphiporus lactifloreus und ihre engere Verwandtschaft entwickelten während ihrer Evolution einen ausstülpbaren Rüssel, der Toxine absondert und mit einer mineralischen Spitze versehen ist, die wie ein Projektil die Körperwand der Beute durchschlägt. Unsere Untersuchungen zur Giftzusammensetzung in A. lactifloreus zeigen, dass proteolytische Enzyme eingebettet in Mucin-Proteine die Grundlage dieses Giftsystems bilden (Abb. 3). Dazu kommen einige vermutlich neurotoxische Komponenten, die Ähnlichkeit mit bekannten Toxinen aus Seesternen (Nemertotoxin-1) und Anemonen (Nemertotoxin-2) haben. Interessanterweise kommen die meisten dieser Substanzen nicht nur im A. lactifloreus-Gift vor, sondern sind auch in der körperbedeckenden Schleimschicht der Tiere vorhanden.
Neben diesen Beispielen arbeiten Mitglieder unserer Gruppe an der Evolution von Giftsystemen einer Vielzahl von vernachlässigten Gifttieren und dem möglichen Anwendungspotenzial ihrer Giftkomponenten. So werden derzeit Fische, weitere Spinnen sowie Raubwanzen, Raubfliegen, Taubwarane und der Feuersalamander bearbeitet.

\section{Zugriff auf neue Bioressourcen durch Biotechnologie}

Ein Hindernis in der Erforschung von Gifttieren mit geringer Körpergröße ist der sehr limitierte Giftvorrat. Dies macht es schwer oder oft unmöglich, genügend Material zu gewinnen, um die Bioaktivität der Giftbestandteile nach einer Fraktionierung zu bestimmen [11]. Das große Potenzial dieser vernachlässigten Giftsysteme für die Wirkstoffforschung ist daher bislang nicht ausgeschöpft.

Die moderne Venomics ändert dies nun, denn zusammen mit der Aufklärung der Giftzusammensetzung wird durch die gekoppelten Transkriptom- und Genomanalysen auch 
die Erbinformation der Toxine entschlüsselt. Die Anwendung biotechnologischer Verfahren der Proteinherstellung ermöglicht ausgehend von dieser Information die Produktion der identifizierten Moleküle ohne größere Mengen Rohgift. Je nach Komplexität und Struktur der Toxine kommen hierfür verschiedenste Herstellungsansätze zum Einsatz. Während für linear gebaute und sehr kurze Peptide meist Synthesechemie eingesetzt wird, werden größere und komplex aufgebaute Komponenten rekombinant in Escherichia coli produziert. In diesen Systemen konnten wir bereits einige Toxine aus Krebstieren, Skorpionen und Raubfliegen herstellen. Die Untersuchung der Bioaktivitäten dieser Substanzen ist derzeit im Gange.

Ein Nachteil dieser Ansätze ist, dass zumindest für die Strategien über E. coli immer genetisch modifizierte Organismen erzeugt werden müssen. Eine vielversprechende Alternative dazu sind zellfreie Proteinsynthese-Technologien. Ein Ziel unserer Gruppe ist es, diese Technologien für die Herstellung von Tiergiften zu implementieren. Für Neurotoxine aus Sandkrabbenspinnen, Ficoline aus Wassertrugnattern und Xibalbinen von Remipeden liegen bereits erste, vielversprechende Ergebnisse vor.

\section{Literatur}

[1] Fry BG, Roelants K, Champagne DE et al. (2009) The toxicogenomic multiverse: convergent recruitment of proteins into animal venoms. Annu Rev Genom Hum Gen 10:483-511 [2] Casewell NR, Wüster W, Vonk F J et al. (2013) Complex cocktails: the evolutionary novelty of venoms. Trends Ecol Evol 28:219-229

[3] Holford M, Daly M, King GF et al. (2018) Venoms to the rescue. Science 361:842-844

[4] Herzig V, King GF, Undheim EAB (2019) Can we resolve the taxonomic bias in spider venom research? Toxicon: $X$ 1:100005

[5] von Reumont BM, Campbell LI, Jenner RA (2014) Quo vadis venomics? A roadmap to neglected venomous invertebrates. Toxins 6:3488-3551

[6] Drukewitz SH, von Reumont BM (2019) The significance of comparative genomics in modern evolutionary venomics. Front Ecol Evol doi: 10.3389/fevo.2019.00163

[7] Özbek R, Wielsch N, Vogel H et al. (2019) Proteo-

transcriptomic characterization of the venom from the endoparasitoid wasp Pimpla turionellae with aspects on its biology and evolution. Toxins 11:721

[8] Özbek R, Mukherjee K, Uçkan F et al. (2020) Reprograming of epigenetic mechanisms controlling host insect immunity and development in response to egg-laying by a parasitoid wasp. Proc Biol 287:20200704

[9] Lüddecke T, von Reumont BM, Förster F et al. (2020) An economic dilemma between molecular weapon systems may explain an arachnological-atypical venom in wasp spiders (Argiope bruennichi). Biomolecules 10:978

[10] von Reumont BM, Lüddecke T, Timm T et al. (2020) Proteo-transcriptomic analysis identifies novel toxins secreted by the predatory, prey piercing ribbon worm Amphiporus lactifloreus. Marine Drugs 18:407

[11] von Reumont BM (2018) Studying smaller and neglected organisms in modern evolutionary venomics implementing RNAseq (transcriptomics) - A Critical Guide. Toxins 10:292

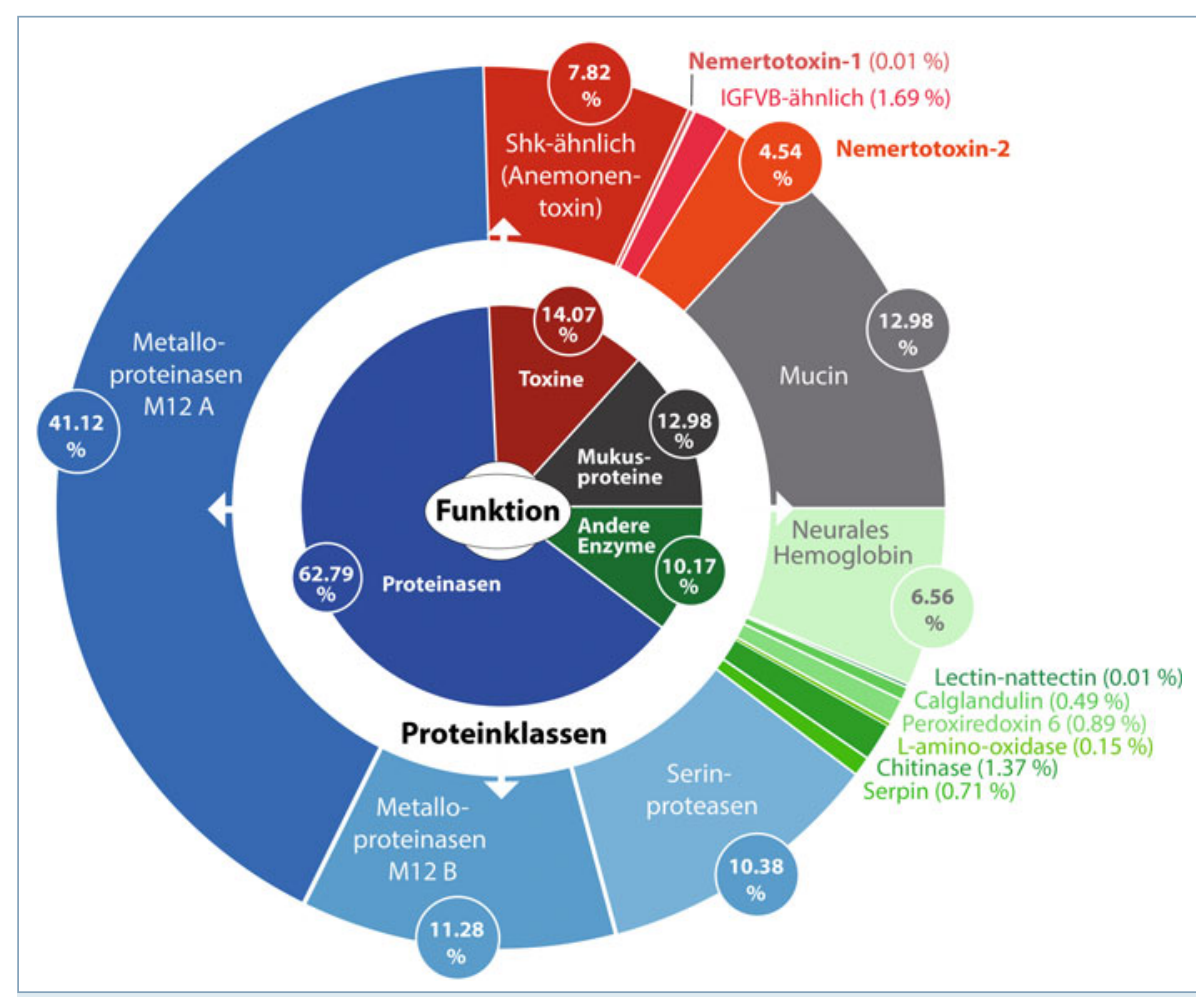

$\Delta$ Abb. 3: Funktionale Übersicht des ersten beschriebenen Giftcocktails von Schnurwürmern für die Nemertinenart $A$. lactifloreus. Die in Rot gehaltenen Giftkomponenten (Toxine) weisen Cysteineabfolgen auf, die für die Klasse der Knottine typisch sind. Diese Peptide bilden robuste Faltungen aus und interagieren mit lonenkanälen, wodurch sie oft neurotoxische Wirkung zeigen.

Funding note: Open Access funding enabled and organized by Projekt DEAL. Open Access: Dieser Artikel wird unter der Creative Commons Namensnennun 4.0 International Lizenzveröffentlicht, welche die Nutzung, Vervielfältigung, Bearbeitung, Verbreitung und Wiedergabe in jeglichem Medium und For erlaubt, sofern Sie den/die ursprünglichen Autor(en) und die Quelle
ordnungsgemäß nennen, einen Link zur Creative Commons Lizenz beifügen und angeben, ob Änderungen vorgenommen wurden. Die in diesem Artike enthaltenen Bilder und sonstiges Drittmaterial unterliegen ebenfalls der genannten Creative Commons Lizenz, sofern sich aus der Abbildungslegen genannten Creative. Sof nas betroter nach gesetzlichen Vorschriften erlaubt ist, ist für die oben aufgeführten nic Weiterverwendungen des Materials die Einwilligung des jeweiligen Rechteinhabers einzuholen. Weitere Details zur Lizenz entnehmen Sie bitte der Lizenzinformation auf http://creativecommons.org/licenses/by/4.0/deed.de.

\section{Korrespondenzadresse:}

Dr. rer. nat. habil. Björn M. von Reumont Institut für Insektenbiotechnologie und LOEWE Zentrum für Translationale Biodiversitätsgenomik

Justus-Liebig-Universität Gießen

Heinrich-Buff-Ring 58

D-35392 Gießen

Bjoern.Von-Reumont@agrar.uni-giessen.de

\begin{tabular}{|c|c|}
\hline \multicolumn{2}{|l|}{ AUTOREN } \\
\hline & $\begin{array}{l}\text { Tim Lüddecke } \\
\text { 2008-2012 Chemielaborant in der Heubach GmbH. 2012-2018 Biologiestudium an } \\
\text { der TU Braunschweig. Seit } 2018 \text { Doktorand am Fraunhofer Institut für Molekularbiolo- } \\
\text { gie und Angewandte Ökologie in Gießen im Bereich Bioprospektion von Spinnengiften. } \\
\text { Seit } 2018 \text { assoziierter Wissenschaftler am LOEWE-Zentrum für Translationale Bio- } \\
\text { diversitätsgenomik (LOEWE-TBG), Frankfurt a. M. Seit } 2019 \text { Vizepräsident der Deut- } \\
\text { schen Arachnologischen Gesellschaft. }\end{array}$ \\
\hline & $\begin{array}{l}\text { Björn M. von Reumont } \\
\text { 1999-2005 Studium der Biologie und 2005-2010 Promotion an der Universität } \\
\text { Bonn. Danach Postdoktorand am Zoologischen Forschungsmuseum König, Bonn, } \\
\text { Natural History Museum, London, UK und Assistent an der Universität Leipzig mit Ha- } \\
\text { bilitation 2020. Seit } 2018 \text { Koordinator der Gruppe Tiergifte am Institut für Insekten- } \\
\text { biotechnologie an der Universität Gießen und dem LOEWE-Zentrum für Translationale } \\
\text { Biodiversitätsgenomik (LOEWE-TBG) in Frankfurt a. M. }\end{array}$ \\
\hline
\end{tabular}

